\title{
Segmentation of 3D Probability Density Fields by Surface Evolution: Application to Diffusion MRI
}

\author{
Christophe Lenglet, Mikaël Rousson, and Rachid Deriche \\ I.N.R.I.A. Sophia Antipolis, France \\ \{clenglet, mrousson, der\}@sophia.inria.fr
}

\begin{abstract}
We propose an original approach for the segmentation of three-dimensional fields of probability density functions. This presents a wide range of applications in medical images processing, in particular for diffusion magnetic resonance imaging where each voxel is assigned with a function describing the average motion of water molecules. Being able to automatically extract relevant anatomical structures of the white matter, such as the corpus callosum, would dramatically improve our current knowledge of the cerebral connectivity as well as allow for their statistical analysis. Our approach relies on the use of the symmetrized Kullback-Leibler distance and on the modelization of its distribution over the subsets of interest in the volume. The variational formulation of the problem yields a level-set evolution converging toward the optimal segmentation.
\end{abstract}

\section{Introduction}

Diffusion magnetic resonance imaging is a relatively new modality [4, 9] able to quantify the anisotropic diffusion of water molecules in highly structured biological tissues. In 1994, P. Basser [2] proposed to model the probability density function of the molecular motion $r \in \mathbb{R}^{3}$ by a Gaussian law whose covariance matrix is given by the diffusion tensor D. Diffusion Tensor Imaging (DTI) then produces a volumic image containing, at each voxel, a $3 \times 3$ symmetric positivedefinite tensor. The estimation of these tensors requires the acquisition of diffusion weighted images in different sampling directions together with a T2 image. Numerous algorithms have been proposed to perform a robust estimation and regularization of these tensors fields [13, [18. Recently, Q-ball Imaging has been introduced by D. Tuch etal. [14] in order to reconstruct the Orientation Distribution Function (ODF) by the Funk-Radon transform of high b-factor diffusion weighted images acquired under the narrow pulse approximation. This ODF is the symmetric probability density function $S^{2} \rightarrow \mathbb{R}$ giving the probability for a spin to diffuse in a given direction. This method provides a better angular constrast and is able to recover intra-voxel fiber crossings.

Diffusion MRI is particularly relevant to a wide range of clinical pathologies investigations such as acute brain ischemia detection [12], stroke, Alzheimer disease, schizophrenia [1] ...etc. It is also extremely useful in order to identify the 
neural connectivity of the human brain [8], 15], [5]. As of today, diffusion MRI is the only non-invasive method that allows us to distinguish the various anatomical structures of the cerebral white matter such as the corpus callosum, the arcuate fasciculus or the corona radiata. These are examples of commisural, associative and projective neural pathways, the three major types of fiber bundles, respectively connecting the two hemispheres, regions of a given hemisphere or the cerebral cortex with subcortical areas. In the past, many techniques have been proposed to classify gray matter, white matter and cephalo-spinal fluid from T1weighted MR [20] images but the literature addressing the issue of white matter internal structures segmentation is just beginning [21, [7, [17, [19].

In the following, we introduce a novel technique for the segmentation of any probability density function $(p d f)$ field by examining the statistics of the distribution of the Kullback-Leibler distances between these $p d f s$. Our goal is to perform the direct segmentation of internal structures of the white matter. Zhukov et al. [21] defined an invariant anisotropy measure in order to drive the evolution of a level-set and isolate strongly anisotropic regions of the brain. The reduction of the full tensor to a single scalar gives a relatively low discrimination power to the method potentially resulting in segmentation of mixed structures. On the other side, Wiegell et al. [19], Jonasson et al. [7] and Wang et al. [16] proposed different measures of dissimilarity between full diffusion tensors: The first method uses the Frobenius norm of the difference of tensors, together with a spatial coherence term in a $k$-means algorithm to perform the segmentation of the thalamus nuclei. The nature of the elements to be segmented (compact, homogeneous) verify the restrictive hypothesis of the technique, which is rarely the case. The second method introduces a geometric measure of dissimilarity by computing the normalized tensor scalar product of two tensors, which can be interpreted as a measure of overlap. Finally, the third method relies on the natural distance between two Gaussian $p d f s$, given by the symmetrized Kullback-Leibler distance. The authors elegantly derive an affine invariant dissimilarity measure between diffusion tensors and apply it to the segmentation of $2 \mathrm{D}$ fields of $p d f s$. We generalize the existing methods to the $3 \mathrm{D}$ case and exploit the information provided by the statistics of the distribution of the symmetrized Kullback-Leibler (KL) distances. KL distances are taken between any $p d f$ and our method is thus applicable not only to DTI but also, for example, to Q-ball data which should enable the proposed algorithm to catch even finer details. Section 2 will derive the evolution equation used to drive a 3D surface toward the optimal segmentation. Section 3 will present and discuss experimental results both on synthetic and real DTI datasets.

\section{Derivation of the Level-Set Evolution}

Let $p(x, r)$ be the probability density function of a random vector $r$ of $\mathbb{R}^{3}$ describing the water molecules average motion at a given voxel $x$ of a diffusion $\mathrm{MR}$ image $\Omega \subset \mathbb{R}^{3}$ and for a given diffusion time $\tau$ imposed by the parameters of the PGSE (Pulsed Gradient Spin Echo) sequence. We are interested in characterizing the global coherence of that $p d f$ field and use the classical symmetrized 
Kullback-Leibler distance to that end. With $p(x), q(y) \forall x, y \in \Omega$ two $p d f s$ from $\mathbb{R}^{3}$ onto $\mathbb{R}^{+}$, their $\mathrm{KL}$ distance is given by

$$
d(p, q)=K L(p, q)=\frac{1}{2} \int_{\mathbb{R}^{3}}\left(p(r) \log \frac{p(r)}{q(r)}+q(r) \log \frac{q(r)}{p(r)}\right) d r
$$

Assuming a partition of the data between the structure we try to segment $\Omega_{1}$ and the rest of the volume $\Omega_{2}$, we seek the optimal separating surface $\Gamma$ between those two subsets. We denote by $\bar{p}_{1}$ and $\bar{p}_{2}$ the most representative $p d f s$ over $\Omega_{1}$ and $\Omega_{2}$ verifying equation 5. It is then possible to model the distribution of the KL distances to $\bar{p}_{1}$ and $\bar{p}_{2}$ in their respective domains by suitable densities $p_{d, 1}, p_{d, 2}$. In the following, we make the assumption that $p_{d, 1}, p_{d, 2}$ are Gaussian of zero mean and variances $\sigma_{1}^{2}, \sigma_{2}^{2}$. It is indeed natural to impose the mean distance to the $p d f s \bar{p}_{1}$ and $\bar{p}_{2}$ to be as small as possible, while retaining an important degree of freedom by considering the variances of those distributions.

We then define the following energy in order to maximize the likelihood of these densities on their associated domain:

$$
E\left(\Omega_{i}, \sigma_{i}^{2}, \bar{p}_{i}\right)=\sum_{i=1}^{2} \int_{\Omega_{i}}-\log p_{d, i}\left(d\left(p(x), \bar{p}_{i}\right)\right) d x
$$

where $p_{d, i}=\frac{1}{\sqrt{2 \pi \sigma_{i}^{2}}} \exp \frac{-d^{2}\left(p, \bar{p}_{i}\right)}{2 \sigma_{i}^{2}}$. We denote by $\phi: \Omega \rightarrow \mathbb{R}^{3}$ the level set distance function whose zero isosurface coincides with $\Gamma$. We define $H_{\epsilon}(z)$ and $\delta_{\epsilon}(z)$ the regularized versions of the Heaviside and Dirac functions [6] and we can now rewrite equation 2 and introduce a regularity constraint on $\Gamma$ as follows:

$$
\int_{\Omega}-\log p_{d, 1}\left(d\left(p(x), \bar{p}_{1}\right)\right) H_{\epsilon}(\phi)-\log p_{d, 2}\left(d\left(p(x), \bar{p}_{2}\right)\right)\left(1-H_{\epsilon}(\phi)\right)+\nu\left|\nabla H_{\epsilon}(\phi)\right| d x
$$

The derivation of the Euler-Lagrange equations for this class of energy was studied in 11 and yields the following evolution for $\phi$ :

$$
\phi_{t}(x)=\delta_{\epsilon}(\phi(x))\left(\nu \operatorname{div} \frac{\nabla \phi}{|\nabla \phi|}+\frac{1}{2} \log \frac{p_{d, 2}}{p_{d, 1}}\right) \forall x \in \Omega
$$

Moreover, the derivation of the energy with respect to $\sigma_{i}^{2}$ and $\bar{p}_{i}$ provides the update formulae for these statistical parameters. It can be shown that the variance must be updated with its empirical estimation, whereas some more work is needed for the $\bar{p}_{i}$. We indeed have to estimate:

$$
\tilde{p}_{i}=\operatorname{argmin} \int_{\Omega_{i}} K L^{2}\left(\bar{p}_{i}, p(x)\right) d x
$$

For a general $p d f p(x)$, for instance if we consider the ODF derived from Q-ball data, the variance is easily computed as in [11 but the estimation of the $\tilde{p}_{i}$ might require the use of numerical approximation techniques if no closed form 
is available. If we now come back to the DTI case (ie. Gaussian $p d f s$ ), a recent paper by Wang et al. [16] nicely showed that the mean value of the tensor field is given by $\tilde{\mathbf{D}}_{i}=\sqrt{\mathbf{B}_{i}^{-1}}\left(\sqrt{\sqrt{\mathbf{B}_{i}} \mathbf{A}_{i} \sqrt{\mathbf{B}_{i}}}\right) \sqrt{\mathbf{B}_{i}^{-1}}$ where $\mathbf{A}_{i}=\int_{\Omega_{i}} \mathbf{D}(x) d x$ and $\mathbf{B}_{i}=\int_{\Omega_{i}} \mathbf{D}^{-1}(x) d x$

\section{Experimental Results and Comparisons}

We begin with a validation of our approach on synthetical data with limit cases where other approaches fail. Then, experiments are conducted on the extraction of the corpus callosum from real DTI data. Finally, we show how we can improve the robustness of our approach by introducing an anisotropy measure.

\subsection{Synthetical Data}

Diffusion tensor images measure the displacement of water molecules. This displacement, which characterizes different tissues, can be split into two different information: its intensity and its direction. When considering diffusion tensor images, these information are given respectively by the largest eigenvalue and the corresponding eigenvector. From this decomposition, we built one limit case where the two regions differ only with respect to the main orientation of the tensors. Moreover, to stress the algorithm, we also impose some degree of variation on the main orientation within the inside region by creating a junction as shown in Fig 1. Last, but not least, a Gaussian noise was added directly on the eigen-elements of each tensor. Initializing the surface with a bounding box, our approach is able to give the expected segmentation (Fig 1).

However, this example does not show the necessity of including a statistical model for the distance distribution of each region and the approach proposed in [16] for $2 \mathrm{D}$ fields of $p d f$ gives a similar result. In order to show the advantages of our model, which is more general, we have generated a second test image. As shown in Fig,2, it is composed by one torus whose internal tensors are oriented according to the tangents of the central radius of the torus. Noise is also added to all the tensors of the image but with a different variance whether the tensor is inside or outside the torus. In Fig, 2, we compare the results obtained using [16] and our approach, for different initializations. The first method fails to segment the torus because of the orientations high variations within each region. If we initialize with a bounding box, the surface shrinks until it disappears and if we start from a small sphere inside the torus, only a small part of the torus can be captured. Using our approach, which models the variance of the tensors, the torus is correctly extracted for the different initializations.

\subsection{Real DTI Data}

Data acquisition: Our dataset consists of 30 diffusion weighted images $S_{k}$ : $\Omega \rightarrow \mathbb{R}, k=1, \ldots, 30$ as well as 1 image $S_{0}$ corresponding to the signal intensity in the absence of a diffusion-sensitizing gradient field. They were obtained on a GE 

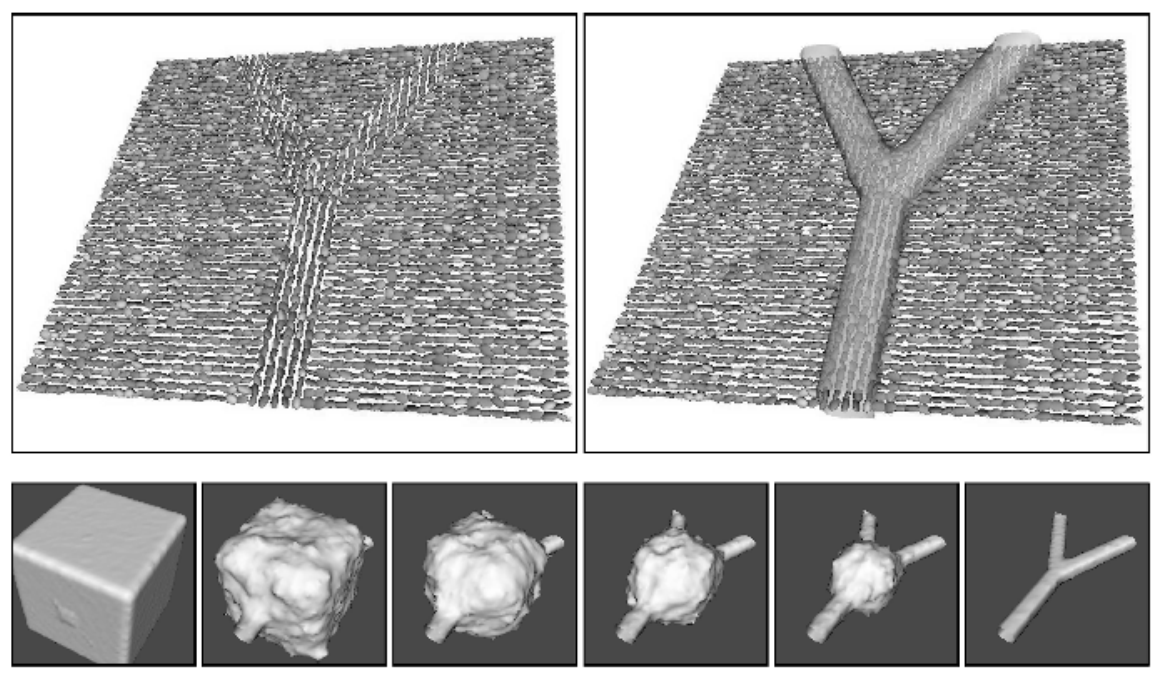

Fig. 1. Segmentation of a noisy tensor field composed by two regions with same scale but different orientations (TOP LEFT: 2D-cut of the tensor field, Top RIGHT: same with final segmentation, Воттом: surface evolution).

1.5 T Signa Echospeed with standard $22 \mathrm{mT} / \mathrm{m}$ gradient field. The echoplanar images were acquired on 56 evenly spaced axial planes with a $128 \times 128$ pixels in each slice. Voxel size is $1.875 \mathrm{~mm} \times 1.875 \mathrm{~mm} \times 2.8 \mathrm{~mm} .6$ gradient directions $\mathbf{g}_{k}$, each with 5 different $b$-factors and 4 repetitions were used. Imaging parameters were: $b$ values between 0 and $1000 \mathrm{~s} . \mathrm{mm}^{-2}, T R=2.5 \mathrm{~s}, T E=84.4 \mathrm{~ms}$ and a square field of view of $24 \mathrm{~cm}$ [10]. Those data are courtesy of CEA-SHFJ/Orsay, France 1 . References on the estimation and the regularization of diffusion tensors can be found in [13].

Experiments: The extraction of anatomical structures from DTI data is of great interest since it gives the opportunity to discriminate structures like the corpus callosum which is much harder to characterize using other modalities. Before any processing, the image is cropped around the element of interest to respect the assumption of bi-partitioning imposed by our model. The first experiment aims at extracting the lateral ventricles. Two small spheres are placed inside the ventricles to initialize the surface. The evolution and the final segmentation are shown in Fig 3. This result looks close to what can be expected from anatomical knowledge.

Improvements using the anisotropy: When we consider only the region terms presented in Section 2, the initialization is really important and in many cases, several seeding points have to be set manually to avoid the surface to get

\footnotetext{
${ }^{1}$ The authors would like to thank J.F. Mangin and J.B Poline for providing us with the data
} 

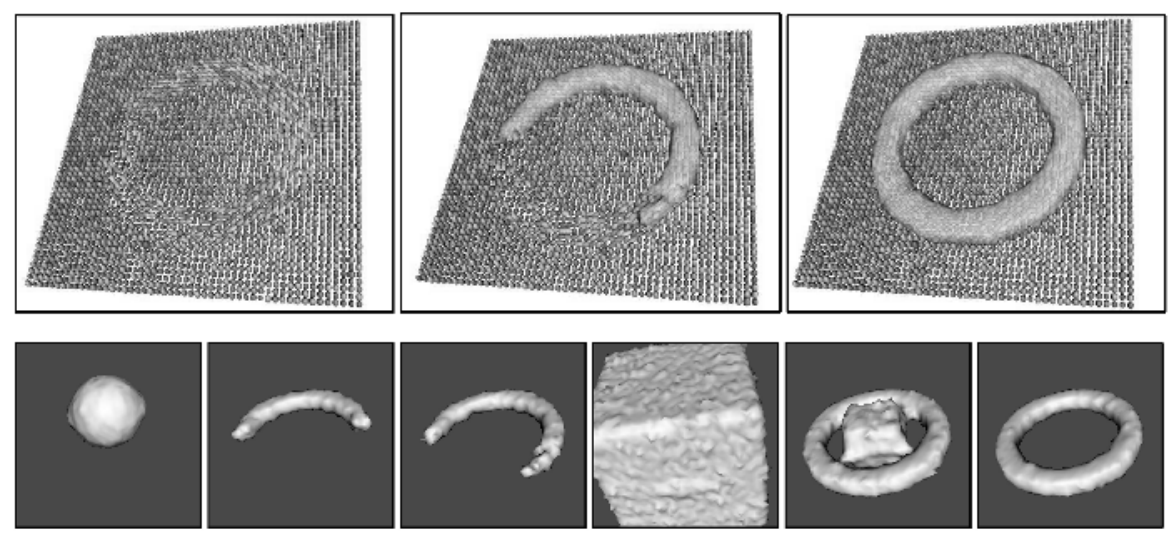

Fig. 2. Segmentation of a noisy tensor field composed by two regions with different orientations (TOP LEFT: 2D-cut of the tensor field, TOP CENTER: segmentation obtained from [16], Top Right: segmentation obtained with our method, Bоттом: surface evolution for both of them).

stuck in a local minima. This can be overcome by introducing a global anisotropy measure. A popular one is the fractional anisotropy [3]:

$$
\mathcal{A}(\mathbf{D}(x))=\frac{\sqrt{\left(\lambda_{1}-\lambda_{2}\right)^{2}+\left(\lambda_{2}-\lambda_{3}\right)^{2}+\left(\lambda_{1}-\lambda_{3}\right)^{2}}}{\sqrt{2} \sqrt{\lambda_{1}^{2}+\lambda_{2}^{2}+\lambda_{3}^{2}}}
$$

An additional term is then defined to impose a given distribution of the anisotropy inside each region. Let $p_{a, 1}$ and $p_{a, 2}$ be the $p d f$ of the anisotropy in $\Omega_{1}$ and $\Omega_{2}$, approximated by Gaussian densities. Then, according to [11] the partitioning is obtained by minimizing:

$$
-\int_{\Omega} \log p_{a, 1}(\mathcal{A}(\mathbf{D}(x))) H_{\epsilon}(\phi)+\log p_{a, 2}(\mathcal{A}(\mathbf{D}(x)))\left(1-H_{\epsilon}(\phi)\right) d x
$$

This term is added to the objective function (3) defined in Section 2. Then, we obtain a new evolution equation for the level set function $\phi$ composed by two terms whose influence can be controlled by adjusting the weight $\alpha$ between zero and one:

$$
\phi_{t}(x)=\delta_{\epsilon}(\phi(x))\left(\nu \operatorname{div} \frac{\nabla \phi}{|\nabla \phi|}+(1-\alpha) \frac{1}{2} \log \frac{p_{d, 2}}{p_{d, 1}}+\alpha \frac{1}{2} \log \frac{p_{a, 2}}{p_{a, 1}}\right) \forall x \in \Omega
$$

and the statistical parameters of $p_{a, 1}$ and $p_{a, 2}$ are updated iteratively like in the previous part. In practice, a small weight on the anisotropy term is sufficient to avoid the surface to get stuck in a local minima. For example, the extraction of the corpus callosum in Fig 3 was possible thanks to this additional term by setting $\alpha$ to 0.3 . 

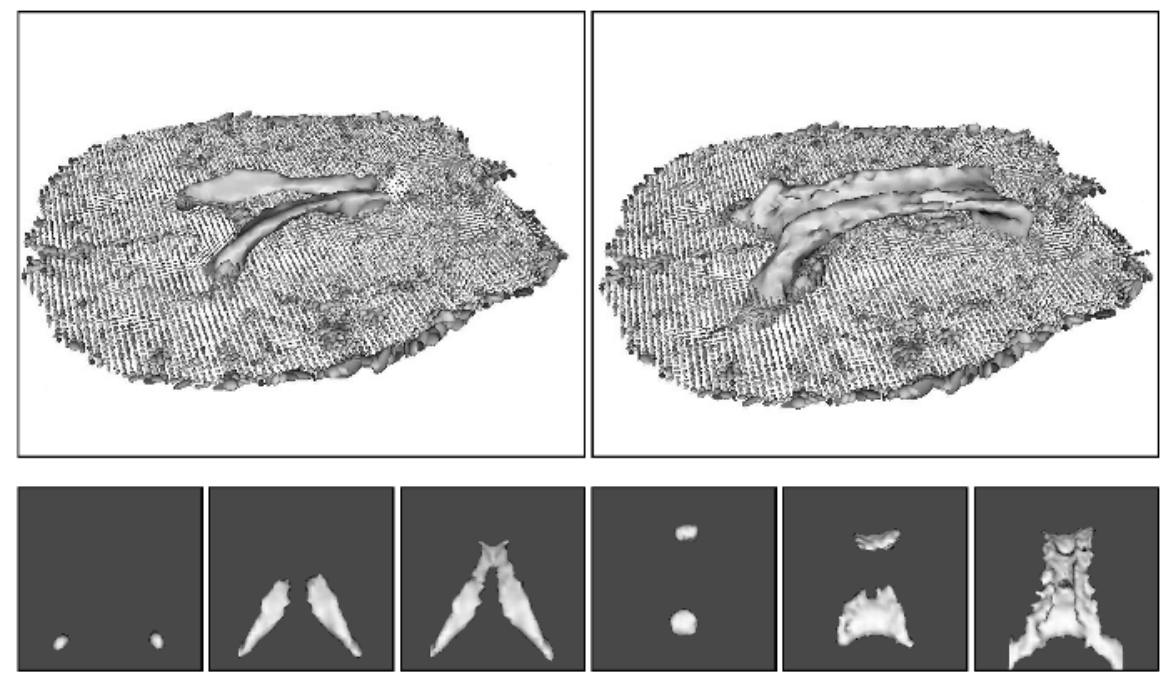

Fig. 3. Segmentation of the brain lateral ventricles (TOP LEFT) and the corpus callosum (TOP Right) in a real diffusion tensor image, superimposed on the DTI field, Воттом: surface evolution for both of them).

\section{Conclusion}

We have presented a novel technique for the segmentation of probability density fields with a major contribution to the extraction of anatomical structures in anisotropic biological tissues such as the brain white matter. We have shown that this method performs very well on synthetic data and is able to catch fine details on real DTI thus exhibiting an adequate behavior of the Kullback-Leibler distance and of the modelization of its distribution by Gaussian densities. We are currently working on general density functions derived, for example, from Q-ball imaging to better describe the voxel-wise diffusion process.

Acknowledgements. The authors would like to thank O. Faugeras (INRIA, Sophia-Antipolis), G. Sapiro, K. Ugurbil, S. Lehericy, K. Lim (University of Minnesota), B. Vemuri and Y. Chen (University of Florida) for their valuable discussions and comments.

\section{References}

1. B.A. Ardekani, J. Nierenberg, M.J. Hoptman, D.C. Javitt, and K.O. Lim. MRI study of white matter diffusion anisotropy in schizophrenia. NeuroReport, 14(16):2025-2029, November 2003.

2. P.J. Basser, J. Mattiello, and D. LeBihan. MR diffusion tensor spectroscopy and imaging. Biophysica, (66):259-267, 1994. 
3. P.J. Basser and C. Pierpaoli. Microstructural and physiological features of tissues elucidated by quantitative diffusion tensor MRI. Journal of Magnetic Resonance, 11:209-219, 1996.

4. D. Le Bihan, E. Breton, D. Lallemand, P. Grenier, E. Cabanis, and M. LavalJeantet. MR imaging of intravoxel incoherent motions: Application to diffusion and perfusion in neurologic disorders. Radiology, pages 401-407, 1986.

5. J.S.W. Campbell, K. Siddiqi, B.C. Vemuri, and G.B Pike. A geometric flow for white matter fibre tract reconstruction. In IEEE International Symposium on Biomedical Imaging Conference Proceedings, pages 505-508, July 2002.

6. T. Chan and L. Vese. An active contour model without edges. In Scale-Space Theories in Computer Vision, pages 141-151, 1999.

7. L. Jonasson, P. Hagmann, X. Bresson, R. Meuli, O. Cuisenaire, and J.P Thiran. White matter mapping in DT-MRI using geometric flows. In EUROCAST, pages 585-596, 2003.

8. C. Lenglet, R. Deriche, and O. Faugeras. Inferring white matter geometry from diffusion tensor MRI: Application to connectivity mapping. In ECCV, 4:127-140, 2004 .

9. K.D. Merboldt, W. Hanicke, and J. Frahm. Self-diffusion NMR imaging using stimulated echoes. J. Magn. Reson., 64:479-486, 1985.

10. C. Poupon. Détection des faisceaux de fibres de la substance blanche pour l'étude de la connectivité anatomique cérébrale. PhD thesis, ENST, December 1999.

11. M. Rousson and R. Deriche. A variational framework for active and adaptative segmentation of vector valued images. In Proc. IEEE Workshop on Motion and Video Computing, pages 56-62, Orlando, Florida, December 2002.

12. C. Sotak. The role of diffusion tensor imaging (DTI) in the evaluation of ischemic brain injury. NMR Biomed., 15:561-569, 2002.

13. D. Tschumperlé and R. Deriche. Variational frameworks for DT-MRI estimation, regularization and visualization. In Proceedings of the 9th International Conference on Computer Vision, Nice, France, 2003.

14. D.S. Tuch, T.G. Reese, M.R. Wiegell, and V.J. Wedeen. Diffusion MRI of complex neural architecture. Neuron, 40:885-895, December 2003.

15. B. Vemuri, Y. Chen, M. Rao, T. McGraw, T. Mareci, and Z. Wang. Fiber tract mapping from diffusion tensor MRI. In 1st IEEE Workshop on Variational and Level Set Methods in Computer Vision, July 2001.

16. Z. Wang and B.C. Vemuri. An affine invariant tensor dissimilarity measure and its application to tensor-valued image segmentation. In IEEE Conference on Computer Vision and Pattern Recognition, Washington, DC., June 2004.

17. Z. Wang and B.C. Vemuri. Tensor field segmentation using region based active contour model. In ECCV, 4:304-315, 2004.

18. Z. Wang, B.C. Vemuri, Y. Chen, and T. Mareci. Simultaneous smoothing and estimation of the tensor field from diffusion tensor MRI. In IEEE Conference on Computer Vision and Pattern Recognition, 1:461-466, 2003.

19. M.R. Wiegell, D.S. Tuch, H.W.B. Larson, and V.J. Wedeen. Automatic segmentation of thalamic nuclei from diffusion tensor magnetic resonance imaging. NeuroImage, 19:391-402, 2003.

20. Y. Zhang, M. Brady, and S. Smith. Segmentation of brain MR images through a hidden markov random field model and the expectation-maximization algorithm. IEEE Transactions on Medical Imaging, 20(1):45, January 2001.

21. L. Zhukov, K. Museth, D. Breen, R. Whitaker, and A.H. Barr. Level set segmentation and modeling of DT-MRI human brain data. Journal of Electronic Imaging, 12:125-133, 2003. 